\title{
miR-205 promotes tumor proliferation and invasion through targeting ESRRG in endometrial carcinoma
}

\author{
NING SU ${ }^{1 *}$, HAIFENG QIU ${ }^{1 *}$, YIFEI CHEN ${ }^{2}$, TINGTING YANG $^{1}$, QIN YAN $^{1}$ and XIAOPING WAN ${ }^{1}$ \\ ${ }^{1}$ Department of Obstetrics and Gynecology, ${ }^{2}$ Central Laboratory, International Peace Maternity and Child Health \\ Hospital of the China Welfare Institute Affiliated to Shanghai Jiao Tong University, Shanghai 200030, P.R. China
}

Received January 28, 2013; Accepted March 19, 2013

DOI: 10.3892/or.2013.2400

\begin{abstract}
Increasing evidence suggests that miR-205 is frequently dysregulated in many types of human cancers, suggesting its important roles in the initiation and progression of cancer. However, the functions of miR-205 in human endometrial endometrioid carcinoma (EEC) are still unknown. In this study, we investigated the expression of miR-205 in both normal endometrium and EEC tissues using TaqMan PCR. Compared to normal tissues, miR-205 was significantly upregulated in EEC $(\mathrm{P}<0.001)$. After transfection of miR-205 inhibitors into Ishikawa cells (or transfection of miR-205 mimics into AN3CA cells), we demonstrated that the cellular proliferation, migration and invasion properties were negatively regulated by miR-205. Moreover, by combination of microRNA target prediction algorithms and luciferase reporter system, we identified estrogen-related receptor- $\gamma$ (ESRRG) as a target of miR-205. In conclusion, we demonstrated frequent upregulation of miR-205 in EEC. In gain-of-function and loss-of-function assays, inhibition of miR-205 reduced cellular proliferation, migration and invasion; vice versa, increased levels of miR-205 led to upregulated cellular proliferation, migration and invasion. Nonetheless, we identified the ESRRG gene to be a novel target, which could be helpful to elucidate mechanisms underlying the tumorigenesis of EEC.
\end{abstract}

Correspondence to: Dr Xiaoping Wan, Department of Obstetrics and Gynecology, International Peace Maternity and Child Health Hospital of the China Welfare Institute, No. 910 Hengshan Road, Shanghai 200030, P.R. China

E-mail: wanxiaoping61@126.com

*Contributed equally

Abbreviations: EEC, endometrial endometrioid carcinoma; ESRRG, estrogen-related receptor- $\gamma$; ER, estrogen receptor; qRT-PCR, quantitative reverse transcription-polymerase chain reaction; MTT, 3-(4,5-dimethylthiazol-2-yl)-2,5-diphenyltetrazolium bromide; 3'UTR, 3'untranslated region

Key words: endometrial endometrioid carcinoma, miR-205, ESRRG, proliferation, invasion

\section{Introduction}

Endometrial cancer is the most common malignancy of the female genital system, and in 2011 it led to an estimated 8,120 deaths in the USA (1). The majority of endometrial cancers $(72 \%)$ are detected at an early stage (stage I-II); however, $28 \%$ of patients have regional or distant metastasis at diagnosis (20\% in stage III and $8 \%$ in stage IV) (2). Endometrial endometrioid carcinoma (EEC) accounts for $80-90 \%$ of the cases of endometrial cancers. Although EEC has a relatively low mortality rate, some tumors are aggressive and insensitive to surgery, chemotherapy or radiation therapy. For patients with localized disease, hysterectomy and bilateral salpingo-oophorectomy remain the primary and most effective treatment. However, the etiology of endometrial cancer remains unclear. Therefore, there is an urgent need for new therapeutic targets and strategies, both of which may be obtained through an increased understanding of the molecular mechanisms involved in endometrial tumorigenesis.

microRNAs (miRNAs) are a group of non-coding nucleic acids which regulate gene expression by facilitating the degradation or translational inhibition of their target mRNAs. miRNAs are reported to play important roles in cancer by regulating basic cellular functions including proliferation, differentiation and cell death $(3,4)$. Recently, it has been revealed that the expression of miR-205 is altered in human $\operatorname{EEC~}(5,6)$; however, the function of miR-205 in EEC remains unclear.

In this study, we investigated the potential roles of miR-205 in EEC. Moreover, we identified estrogen-related receptor- $\gamma$ (ESRRG) to be a novel target of miR-205.

\section{Materials and methods}

Tissue collection. Fifty-three samples of endometrial endometrioid carcinoma were obtained from patients who underwent surgical therapy at the International Peace Maternity and Child Health Hospital of the China Welfare Institute from February 2008 to March 2011. The stages and histological grades of these tumors were established according to the FIGO criteria (2009) (7). The characteristics of the patients are shown in Table I. Twenty-two normal endometrial samples were obtained from patients who underwent hysterectomy to treat other diseases including uterine myoma and 
Table I. Correlation between miR-205 expression and clinicopathological variables of the endometrial endometrioid carcinoma cases.

\begin{tabular}{|c|c|c|c|c|c|}
\hline \multirow[b]{2}{*}{ Clinicopathological data } & \multicolumn{2}{|c|}{ No. of patients (\%) } & \multicolumn{2}{|c|}{ miR-205 expression } & \multirow[b]{2}{*}{ P-value ${ }^{a}$} \\
\hline & $\mathrm{n}$ & $\%$ & No. of low (\%) & No. of high (\%) & \\
\hline \multicolumn{6}{|l|}{ FIGO stage } \\
\hline Stage I & 41 & 77.4 & 23 & 18 & 0.496 \\
\hline Stages II and III & 12 & 22.6 & 8 & 4 & \\
\hline \multicolumn{6}{|l|}{ Grade } \\
\hline G1 & 25 & 66.0 & 14 & 6 & 0.246 \\
\hline $\mathrm{G} 2 / 3$ & 28 & 34.0 & 15 & 14 & \\
\hline \multicolumn{6}{|l|}{ Myometrial invasion } \\
\hline$<1 / 2$ & 40 & 75.5 & 22 & 18 & 0.277 \\
\hline$\geq 1 / 2$ & 13 & 24.5 & 7 & 2 & \\
\hline \multicolumn{6}{|l|}{ Nodal metastasis } \\
\hline Negative & 49 & 92.5 & 29 & 20 & 0.162 \\
\hline Positive & 4 & 7.5 & 2 & 2 & \\
\hline \multicolumn{6}{|l|}{ Estrogen receptor status } \\
\hline Negative & 9 & 17.0 & 5 & 4 & 0.358 \\
\hline Positive & 44 & 83.0 & 26 & 18 & \\
\hline
\end{tabular}

${ }^{\mathrm{a} F i s h e r ' s ~ e x a c t ~ t e s t . ~}$

adenomyosis. The research project was approved by the local ethics committee, and informed consent for the experimental use of the surgical samples was obtained from all patients.

RNA isolation and quantitative reverse transcriptionpolymerase chain reaction ( $q R T-P C R)$. Total RNA was extracted using TRIzol reagent (Invitrogen Life Technologies, Carlsbad, CA, USA). The expression of miR-205 was quantified by qRT-PCR using TaqMan microRNA assays (Applied Biosystems, Carlsbad, CA, USA) and normalized to U6B. The expression of ESRRG was quantified by qRT-PCR using SYBR-Green assays (Applied Biosystems) and normalized to $\beta$-actin. The primers used for qRT-PCR are listed in Table II. Gene expression was calculated using the $2^{-\Delta C t}$ method (8).

Cell culture and transfection. The human endometrial carcinoma cell lines Ishikawa, KLE and AN3CA, and human embryonic kidney 293T cells were obtained from the American Type Culture Collection (ATCC, Manassas, VA, USA). All cells were maintained in Dulbecco's modified Eagle's medium (DMEM)/F12 media (Gibco, Auckland, NZ, USA) supplemented with $10 \%$ fetal bovine serum (Biowest, Nuaillé, France) at $37^{\circ} \mathrm{C}$ in $5 \% \mathrm{CO}_{2}$.

The miR-205 mimics, miR-205 inhibitors and negative control molecules were synthesized by GenePharma Co., Ltd. (Shanghai, China), added to culture media at a final concentration of $100 \mathrm{nM}$ and transfected into cells using Lipofectamine ${ }^{\mathrm{TM}} 2000$ (Invitrogen Life Technologies) according to the manufacturer's instructions.

3-(4,5-Dimethylthiazol-2-yl)-2,5-diphenyltetrazolium bromide (MTT) assay. Ishikawa (or AN3CA) cells were seeded in 96-well plates $\left(4 \times 10^{3}\right.$ cells/well) and transfected with miR-205 inhibitor (or miR-205 mimics) duplexes using Lipofectamine ${ }^{\mathrm{TM}} 2000$. After overnight incubation, the media were removed and cell proliferation was evaluated using the MTT assay as previously described (9).

Wound healing assay. Ishikawa (or AN3CA) cells were seeded in 6 -well plates $\left(4 \times 10^{5}\right.$ cells/well) the day before transfection, and then transfected with miR-205 inhibitor (or miR-205 mimics) duplexes using Lipofectamine ${ }^{\mathrm{TM}}$ 2000. The wound healing assay was performed $24 \mathrm{~h}$ post-transfection as previously described (10).

Transwell migration assay. Ishikawa (or AN3CA cells) $\left(5 \times 10^{4} /\right.$ well $)$ were placed in serum-free medium in the top chamber of Transwell migration chambers (Corning, Inc., Corning, NY, USA). After a $24 \mathrm{~h}$ incubation at $37^{\circ} \mathrm{C}$, the cells adhering to the lower membrane were fixed in $4 \%$ paraformaldehyde at room temperature for $20 \mathrm{~min}$ and then stained with crystal violet staining solution (Beyotime Institute Biotech, Shanghai, China) overnight. The stained cells were visualized under a microscope and counted.

Western blotting. Proteins were separated on $10 \%$ SDS-PAGE gels, transferred to PVDF membranes, blocked with 5\% non-fat milk and incubated with the mouse anti-human ESRRG monoclonal antibody (R\&D Systems, Minneapolis, MN, USA) or mouse anti- $\beta$-actin monoclonal antibody (Proteintech Group, Chicago, IL, USA). Immunoreactivity was visualized using SuperSignal West Pico Chemiluminescent Substrate (Thermo Scientific, Rockford, IL, USA) and Kodak XAR-5 film (Sigma-Aldrich, St. Louis, MO, USA). 
Table II. Primers used in the study.

\begin{tabular}{lll}
\hline Identifier & \multicolumn{1}{c}{ Sense primer sequences } & Antisense primer sequences \\
\hline $\begin{array}{l}\text { miR-205 inhibitor } \\
\text { miR-205 inhibitor } \\
\text { (negative control) }\end{array}$ & 5'-CAGACUCCGGUGGAAUGAAGGA-3' (a) & \\
miR-205 mimics & 5'-UCCUUCAUUCCACCGGAGUCUG-3' & 5'-GACUCCGGUGGAAUGAAGAAUU-3' \\
miR-205 mimics & 5'-UUCUCCGAACGUGUCACGUTT-3' (b) & 5'-ACGUGACACGUUCGGAGAATT-3' \\
(negative control) & & \\
ESRRG & 5'-CTACCCTTCTGCTCCTATCCTG-3' & 5'-AGCGATGTCACCACACACTAAA-3' \\
$\beta$-actin & 5'-CAGCCATGTACGTTGCTATCCAGG-3' & 5'-AGGTCCAGACGCAGGATGGCATG-3' \\
ESRRG 3'UTR & 5'-CGGactagtGATGGAAGCCAGCCCTGCCA-3' & 5'-AGGgtttaaacGGCTCACAGCTCCTTCTGAGGC-3'
\end{tabular}

a2'-O-methyl (2'-OMe) modification; 'FAM modification. Restriction endonuclease site are shown as lower case letters.

A

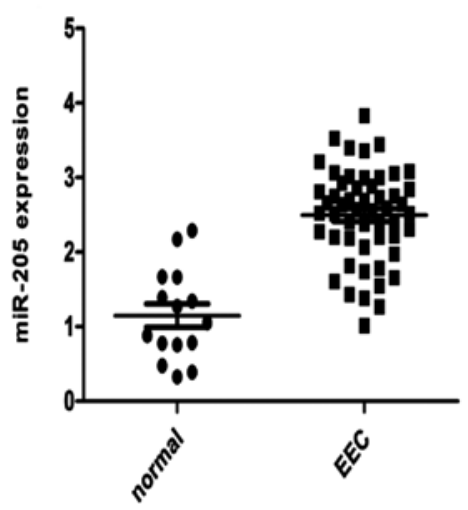

B

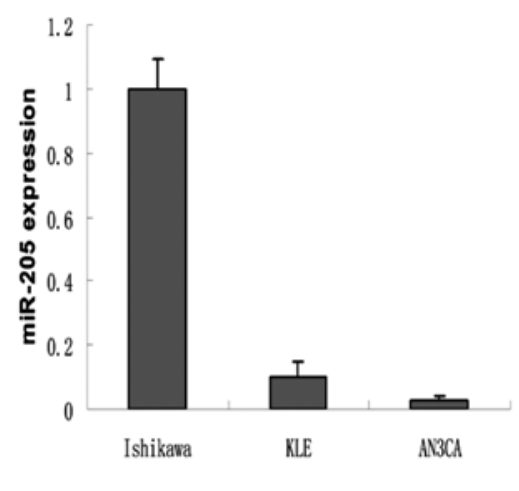

Figure 1. (A) Expression of miR-205 in normal endometrium and EEC cases. (B) Relative expression of miR-205 in the three EEC cell lines.

Construction of reporter plasmids and luciferase assays. The partial 3' untranslated region (3'UTR) of human ESRRG mRNA was cloned between the SpeI and PmeI sites of the pMIR-REPORT ${ }^{\mathrm{TM}}$ vector (Applied Biosystems). The length cloned was 869 bp. A 60-bp mutant ESRRG 3'UTR was also cloned into the pMIR-REPORT ${ }^{\mathrm{TM}}$ vector, which was mutated by the principle of complementary base pairing to disrupt the miR-205 binding sites.

The day prior to transfection, 293T cells were seeded in 96-well plates ( $4 \times 10^{3}$ cells/well) to achieve $\sim 70 \%$ confluency. The cells were co-transfected with pMIR-REPORT ${ }^{\mathrm{TM}}$ constructs containing the wild-type or mutated ESRRG 3'UTR (50 ng/well), pRL-SV40 Renilla luciferase (5 ng/well) and miR-205 mimics (38 nM/well). The luciferase assay was performed $48 \mathrm{~h}$ later as previously described (11).

Statistical analysis. All values are presented as means $\pm \mathrm{SE}$ where appropriate. Categorical data were analyzed using the $\chi^{2}$ test. Quantitative values were evaluated by t-tests or one-way ANOVA using SPSS 16.0 (SPSS, Chicago, IL, USA); $\mathrm{P}<0.05$ was considered to indicate a statistically significant result.

\section{Results}

MiR-205 is overexpressed in EEC tissues. We found that miR-205 was significantly overexpressed in EEC samples when compared with that in the normal control $\left(\chi^{2}=7.146\right.$, $\mathrm{P}=0.008$ ) (Fig. 1). miR-205 was also expressed at high levels in Ishikawa, moderate levels in KLE, and very low levels in AN3CA cells (Fig. 1B).

We analyzed the relationship between miR-205 and FIGO stage, histological grade, myometrial invasion, nodal metastasis and estrogen receptor (ER) status in the EEC cases. However, no association was observed between the expression of miR-205 and these clinicopathological features of the EEC cases (Table I).

Inhibition of miR-205 suppresses cellular proliferation, migration and invasion in Ishikawa cells. We transfected the miR-205 inhibitors into Ishikawa cells for loss-of-function assays. The expression of miR-205 was significantly reduced $72 \mathrm{~h}$ after transfection (Fig. 2A). The MTT assay demonstrated that inhibition of miR-205 inhibited the growth of Ishikawa cells at 24, 48 and $72 \mathrm{~h}$ (Fig. 2B). Downregulation of miR-205 also significantly reduced the migratory and invasive abilities of Ishikawa cells (Fig. 2C and D).

Restoration of miR-205 promotes proliferation, migration and invasion of AN3CA cells. We transfected the miR-205 mimics into AN3CA cells for the gain-of-function assays. After transfection, AN3CA cells showed enhanced proliferation, migration and invasion properties (Fig. 3). 
A

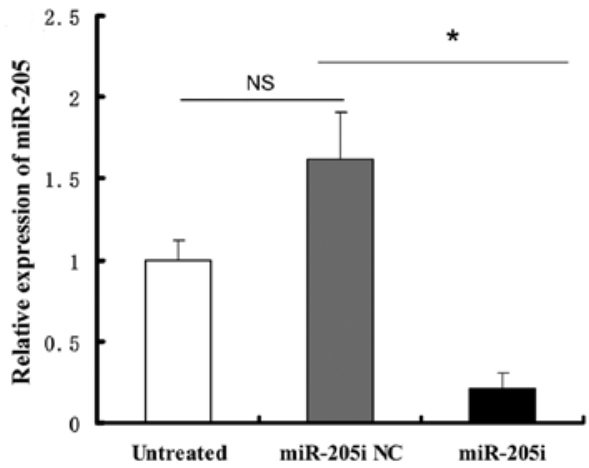

C

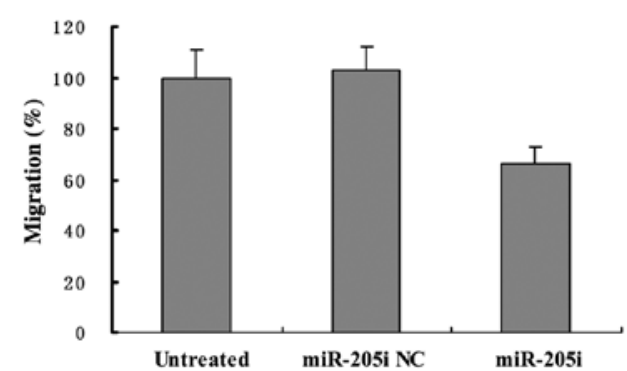

B

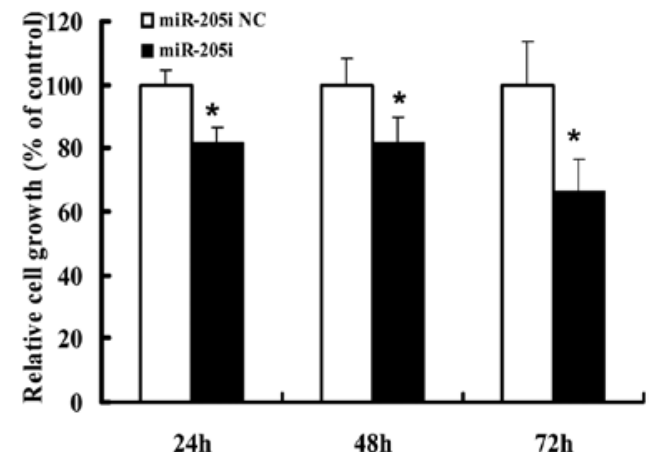

$\mathbf{D}$

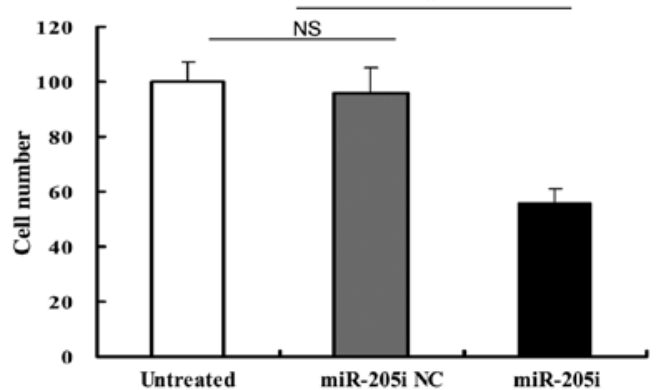

Figure 2. (A) Relative expression of miR-205 in Ishikawa cells $72 \mathrm{~h}$ after transfection ( $\left.{ }^{*} \mathrm{P}<0.05\right)$. (B) MTT assay. The growth of the Ishikawa cells transfected with the miR-205 inhibitor (miR-205i) was significantly inhibited (" $\mathrm{P}<0.01)$. (C) Wound healing assay. The migratory ability of the cells transfected with miR-205i was significantly inhibited ("P<0.01). (D) Transwell assay. Cells transfected with the miR-205i were less invasive than that of the controls ("P<0.01). NS, not significant.

A

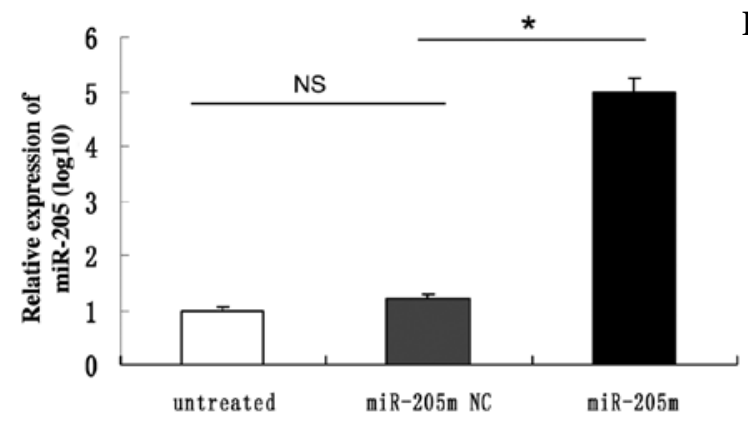

C

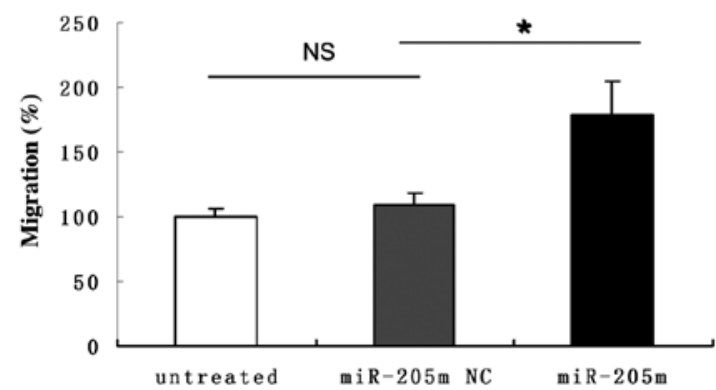

B

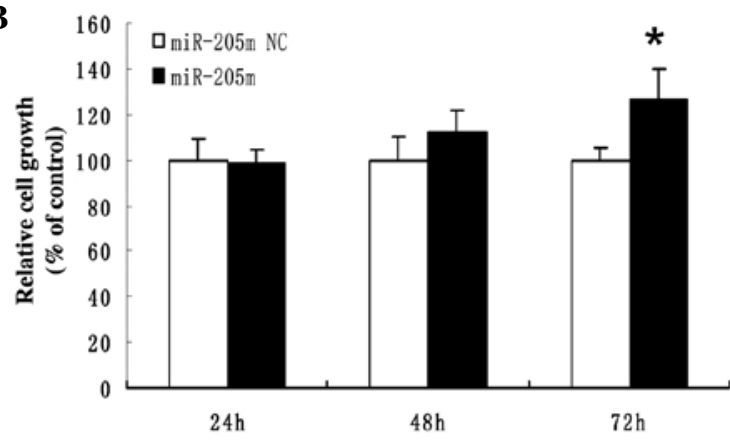

$\mathbf{D}$

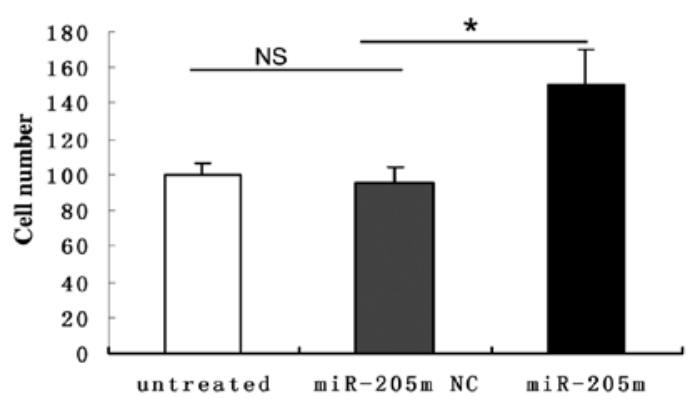

Figure 3. (A) Relative expression of miR-205 in AN3CA cells after transfection ("P<0.05). (B) MTT assay. The growth of AN3CA cells transfected with the miR-205 mimics (miR-205m) was increased to a great extent at $72 \mathrm{~h}\left({ }^{*} \mathrm{P}<0.05\right)$. (C) Wound healing assay. The migration of the cells transfected with the miR-205m was significantly increased ("P<0.01). (D) Transwell assay. The cells transfected with the miR-205m exhibited increased invasive ability than the control cells $($ P $<0.05)$. NS, not significant.

miR-205 directlytargets ESRRG.HumanESRRG was predicted to be a target of miR-205 by TargetScanHuman 6.0 (12). Additionally, ESRRG mRNA and protein expression was negatively correlated with miR-205 levels in the EEC cell lines (Fig. 4).
To determine whether miR-205 regulates expression of ESRRG via the miR-205 binding sites in its 3'UTR, we cloned a fragment of the ESRRG 3'UTR containing the two predicted miR-205 binding sites into a luciferase reporter vector to generate ESRRG_wt (Fig. 5A). The luciferase activity of 
A

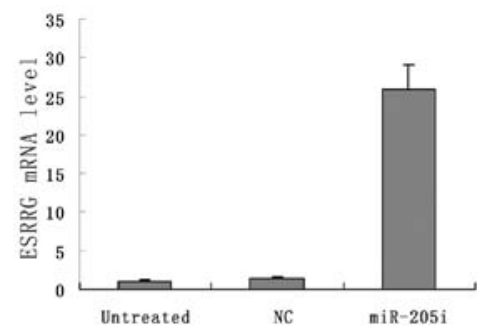

C

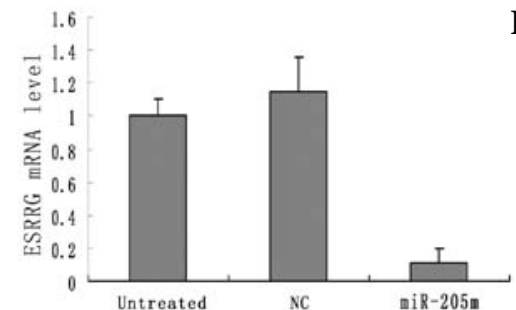

B

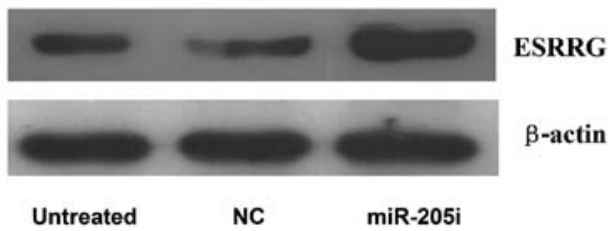

$\mathbf{D}$

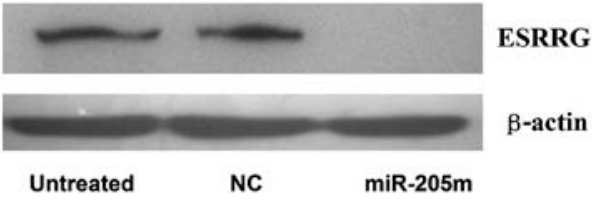

Figure 4. (A) mRNA and (B) protein levels of ESRRG were upregulated after transfection with miR-205 inhibitors (miR-205i). (C) mRNA and (D) protein levels of ESRRG were reduced by transfection with miR-205 mimics (miR-205m). ESRRG, estrogen-related receptor- $\gamma$.

A

Human ESRRG(ERR $\gamma)$ 3'UTR 869nt

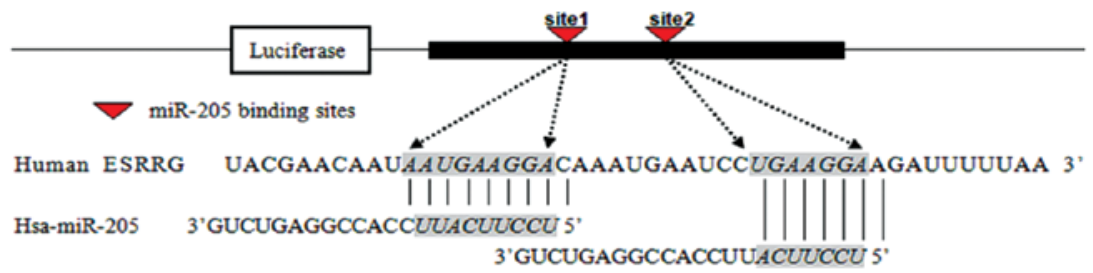

Human UACGAACAAUAAUGAAGGACAAAUGAAUCCUGAAGGAAGAUUUUUAA 3' Chimpanzee UACGAACAAUAAUGAAGGACAAAUGAAUCCUGAAGGAAGAUUUUUAA 3' Rhesus UAUGAACAAUAAUGAAGGACAAAUGAAUCCUGAAGGAAGAUUUUUAAA 3. Mouse U--.-GAACACUAAUGAAGGACAAAUGAACCCUGAAGGAAGAUUUUUAA 3' Rat U.--GAACACUAAUGAAGGACAAAUGAACCCUGAAGGAAGAUUUUUAA 3*

B

$\begin{array}{ll}\text { ESRRG_wt } & \text { UACGAACAAUAAUGAAGGACAAAUGAAUCCUGAAGGAAGAUUUUUAA 3' } \\ \text { ESRRG_mut1 } & \text { UACGAACAAUUUACUUCCUCAAAUGAAUCCUGAAGGAAGAUUUUUAA 3, } \\ \text { ESRRG_mut2 } & \text { UACGAACAAUAAUGAAGGACAAAUGAAUCCACUUCCUAGAUUUUUAA 3, } \\ \text { ESRRG mut3 } & \text { UACGAACAAUUUACUUCCUCAAAUGAAUCCACUUCCUAGAUUUUUAA 3, }\end{array}$

C $\square_{\mathrm{miR}-205 \mathrm{~m} \mathrm{NC}}$

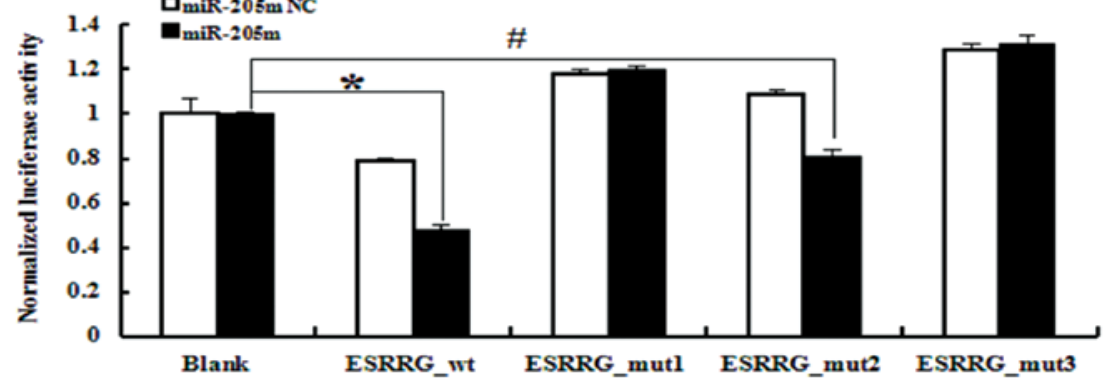

Figure 5. (A) Illustration of the predicted miR-205 binding sites (red arrowheads) in the ESRRG 3'UTR and construction of the pmiR-ESRRG 3'UTR reporter plasmids. Shaded areas represent the conserved complementary nucleotides of the miR-205 seed sequence in selected mammals. (B) The seed regions of the miR-205-binding sites were mutated to disrupt miRNA-target binding (mutated nucleotides are shown in yellow and green). Schematic illustration of the reporter constructs showing the entire 3'UTR ESRRG sequence (ESRRG_wt) and the mutated miR-205 binding sites in the ESRRG 3'UTR (yellow and green) of ESRRG_mut1,ESRRG_mut2 and ESRRG_mut3. (C) Luciferase reporter gene activity of ESRRG_wt and the mutated ESRRG_mut reporter genes in miR-205 mimic-transfected 293T cells (miR-205m) or control transfected cells (miR-205m NC). $\left({ }^{\#} \mathrm{P}<0.05\right.$ and $\left.{ }^{*} \mathrm{P}<0.01\right)$.

ESRRG_wt was significantly reduced $(\sim 50 \%)$ in $293 \mathrm{~T}$ cells treated with the miR-205 mimics (Fig. 5C) $(\mathrm{P}<0.01)$, indicating that miR-205 targets ESRRG via the predicted miR-205 binding sites in the ESRRG 3'UTR.

As the 3'UTR of ESRRG mRNA contains two putative miR-205-binding sites, we mutated the miRNA-binding seed regions of these sites to prevent miRNA binding and created ESRRG_mut1, ESRRG_mut2 and ESRRG_mut3 (Fig. 5B). When the first miR-205-binding site was mutated (ESRRG_mut1) or both miR-205-binding sites were mutated (ESRRG_mut3), the ability of miR-205 mimics to silence reporter gene expression was completely inhibited (Fig. 5C). Mutation of the second miR-205-binding site (ESRRG_mut2) had no effect, suggesting that the first one is the real binding 
site for miR-205 (Fig. 5C). Collectively, these results indicate that ESRRG is a direct target gene of miR-205.

\section{Discussion}

Recent studies have indicated that miR-205 is dysregulated in many types of cancer, such as head and neck cancer (13), squamous cell carcinoma (11) and bladder cancer (14). By TaqMan PCR, we observed that miR-205 was upregulated in EEC compared to normal endometrium $(\mathrm{P}<0.001)$, as well as in the two EEC cell lines, Ishikawa and KLE.

In the gain- and loss-of-function assay, our data indicated that downregulation of miR-205 suppressed the proliferation and migration of Ishikawa cells; conversely, upregulation of miR-205 promoted these properties of AN3CA cells. Therefore, we suggest that miR-205 plays a role as an oncomiR in EEC.

To date, many genes have been identified to be targets of miR-205, including the tumor suppressors SHIP2 (11) and MED1 (15), the oncogenes E2F1, E2F5 and PKCE (16), and the pro-metastatic genes Zeb1 and Zeb2 (17). By microRNA target prediction software, we proposed ESRRG as a target of miR-205, which was confirmed in the luciferase reporter assays. These results suggest that miR-205 acts as an oncomiR through targeting ESRRG.

ESRRG (ERR $\gamma)$, a member of the estrogen-related receptors (ERRs), shares a significant homology with the classical estrogen receptors (ERs) at the amino acid level, and is constitutively activated even in the absence of estrogen. Recently, ESRRG was shown to function as a tumor suppressor in several types of cancers. Tiraby et al reported that increased expression of ESRRG upregulated E-cadherin, promoted the mesenchymal-to-epithelial transition and suppressed breast cancer growth in vivo (18). Likewise, transfection of ESRRG into the LnCaP and DU145 prostate cancer cell lines significantly suppressed proliferation in vitro and tumorigenicity in vivo (19). In the present study, inhibition of miR-205 increased the protein expression of ESRRG, and suppressed cell proliferation, migration and invasion.

In summary, we found frequent upregulation of miR-205 in EEC. In the gain- and loss-of-functions assays, inhibition of miR-205 reduced cellular proliferation, migration and invasion; conversely, upregulated levels of miR-205 led to increased cellular proliferation, migration and invasion. We identified the ESRRG gene to be a novel target, which could be helpful to elucidate mechanisms underlying the tumorigenesis of EEC.

\section{Acknowledgements}

This study was supported by grants from the National Natural Science Foundation of China (nos. 30872760 and 81072139). We would like to thanks Dr Wei Bao, Wen Lu and Guiqiang Du for the technical assistance and Cong Lu and Yuhong Xia for manuscript revision.

\section{References}

1. Siegel R, Ward E, Brawley O and Jemal A: Cancer statistics, 2011: the impact of eliminating socioeconomic and racial disparities on premature cancer deaths. CA Cancer J Clin 61: 212-236, 2011.

2. De Souza Nunes LS, De Oliveira RV, Holgado LA, Nary Filho H, Ribeiro DA and Matsumoto MA: Use of bovine hydroxyapatite with or without biomembrane in sinus lift in rabbits: histopathologic analysis and immune expression of core binding factor 1 and vascular endothelium growth factor. J Oral Maxillofac Surg 69: 1064-1069, 2011.

3. Miska EA: How microRNAs control cell division, differentiation and death. Curr Opin Genet Dev 15: 563-568, 2005.

4. Esquela-Kerscher A and Slack FJ: Oncomirs - microRNAs with a role in cancer. Nat Rev Cancer 6: 259-269, 2006.

5. Chung TK, Cheung TH, Huen NY, Wong KW, Lo KW, Yim SF, Siu NS, Wong YM, Tsang PT, Pang MW, Yu MY, To KF, Mok SC, Wang VW, Li C, Cheung AY, Doran G, Birrer MJ, Smith DI and Wong YF: Dysregulated microRNAs and their predicted targets associated with endometrioid endometrial adenocarcinoma in Hong Kong women. Int J Cancer 124: 1358-1365, 2009.

6. Wu W, Lin Z, Zhuang $\mathrm{Z}$ and Liang $\mathrm{X}$ : Expression profile of mammalian microRNAs in endometrioid adenocarcinoma. Eur J Cancer Prev 18: 50-55, 2009.

7. Creasman W: Revised FIGO staging for carcinoma of the endometrium. Int J Gynaecol Obstet 105: 109, 2009.

8. Schmittgen TD and Livak KJ: Analyzing real-time PCR data by the comparative C(T) method. Nat Protoc 3: 1101-1108, 2008

9. Lee JW, Choi CH, Choi JJ, Park YA, Kim SJ, Hwang SY Kim WY, Kim TJ, Lee JH, Kim BG and Bae DS: Altered microRNA expression in cervical carcinomas. Clin Cancer Res 14: 2535-2542, 2008.

10. Jiang F, Liu T, He Y, Yan Q, Chen X, Wang H and Wan X: MiR-125b promotes proliferation and migration of type II endometrial carcinoma cells through targeting TP53INP1 tumor suppressor in vitro and in vivo. BMC Cancer 11: 425, 2011.

11. Yu J, Ryan DG, Getsios S, Oliveira-Fernandes M, Fatima A and Lavker RM: microRNA-184 antagonizes microRNA-205 to maintain SHIP2 levels in epithelia. Pro Natl Acad Sci USA 105: 19300-19305, 2008.

12. Lewis BP, Shih IH, Jones-Rhoades MW, Bartel DP and Burge CB: Prediction of mammalian microRNA targets. Cell 115: 787-798, 2003.

13. Jiang J, Lee EJ, Gusev Y and Schmittgen TD: Real-time expression profiling of microRNA precursors in human cancer cell lines. Nucleic Acids Res 33: 5394-5403, 2005.

14. Gottardo F, Liu CG, Ferracin M, Calin GA, Fassan M, Bassi P, Sevignani C, Byrne D, Negrini M, Pagano F, Gomella LG, Croce CM and Baffa R: Micro-RNA profiling in kidney and bladder cancers. Urol Oncol 25: 387-392, 2007.

15. Mouillet JF, Chu T, Nelson DM, Mishima T and Sadovsky Y: MiR-205 silences MED1 in hypoxic primary human trophoblasts. FASEB J 24: 2030-2039, 2010.

16. Gandellini P, Folini M, Longoni N, Pennati M, Binda M, Colecchia M, Salvioni R, Supino R, Moretti R, Limonta P, Valdagni R, Daidone MG and Zaffaroni N: miR-205 exerts tumor-suppressive functions in human prostate through downregulation of protein kinase Cepsilon. Cancer Res 69: 2287-2295, 2009.

17. Gregory PA, Bert AG, Paterson EL, Barry SC, Tsykin A, Farshid G, Vadas MA, Khew-Goodall Y and Goodall GJ: The miR-200 family and miR-205 regulate epithelial to mesenchymal transition by targeting ZEB1 and SIP1. Nat Cell Biol 10: 593-601, 2008.

18. Tiraby C, Hazen BC, Gantner ML and Kralli A: Estrogen-related receptor gamma promotes mesenchymal-to-epithelial transition and suppresses breast tumor growth. Cancer Res 71: 2518-2528, 2011.

19. Yu S, Wang X, Ng CF, Chen S and Chan FL: ERR $\gamma$ suppresses cell proliferation and tumor growth of androgen-sensitive and androgen-insensitive prostate cancer cells and its implication as a therapeutic target for prostate cancer. Cancer Res 67: 4904-4914, 2007. 